\title{
Comparative Analysis of Quantitative Mass Spectrometric Methods for Subcellular Proteomics
}

Abla Tannous, Marielle Boonen, Haiyan Zheng, Caifeng Zhao, Colin J. Germain, Dirk F. Moore, David E. Sleat, Michel Jadot, Peter Lobel

\section{SUPPLEMENTAL FIGURES}

Fig. S1. Distribution of precursors with weighted idotp.

Fig. S2-S4. PCA analysis of peptides using all four quantitative proteomics methods.

Table S1. Instrument settings for TMT-MS2 and TMT-MS3 in ML-rate zonal (XLSX)

Table S2. Data acquisition methods for differential centrifugation/Nycodenz fractions. (XLSX)

Table S3. Data acquisition methods for ML-rate zonal fractions (XLSX)

Table S4. Quantification data for TMT-MS2 spectra from differential-Nycodenz experiment after normalization, filtering for recovery and constraining to a common scale so that the values of fractions sum to 1 (XLSX)

Table S5. Quantification data for TMT-MS3 spectra from differential-Nycodenz experiment after normalization, filtering for recovery and constraining to a common scale so that the values of fractions sum to 1 $(\mathrm{XLSX})$

Table S6. Quantification data for MS1 precursors from differential-Nycodenz experiment after normalization, filtering for recovery and constraining to a common scale so that the values of fractions sum to 1 (XLSX) Table S7. Quantification data for DIA elution groups from differential-Nycodenz experiment after normalization, filtering for recovery and constraining to a common scale so that the values of fractions sum to 1 (XLSX)

Table S8. TMT-MS2: Mean protein classification coefficients after outlier rejection (XLSX)

Table S9. TMT-MS3: Mean protein classification coefficients after outlier rejection (XLSX) 
Table S10. MS1: Mean protein classification coefficients after outlier rejection (XLSX)

Table S11. DIA: Mean protein classification coefficients after outlier rejection (XLSX)

Table S12. MetaMass quality metrics for analysis of differential-Nycodenz fractionation by TMT-MS2 \& location assignment (XLSX)

Table S13. MetaMass quality metrics for analysis of differential-Nycodenz fractionation by TMT-MS3 \& location assignment (XLSX)

Table S14. MetaMass quality metrics for analysis of differential-Nycodenz fractionation by MS1 \& location assignment (XLSX)

Table S15. MetaMass quality metrics for analysis of differential-Nycodenz fractionation by DIA \& location assignment (XLSX)

Table S16-Summary MetaMass classification of non-marker proteins \& pairwise comparison of MS methods (XLSX)

Table S17. Markers list used for MetaMass analysis (XLSX)

Table S18. Number of markers proteins per organelle used for MetaMass analysis. (XLSX)

Table S19. Sources \& methods for obtaining marker list (XLSX)

Table S20. Statistics of assigned proteins and peptides of ML-rate zonal (analyzed with differential/Nycodenz ) (XLSX)

Table S21. Statistics of assigned proteins and peptides of Differential/Nycodenz (analyzed with ML-rate zonal) (XLSX) 


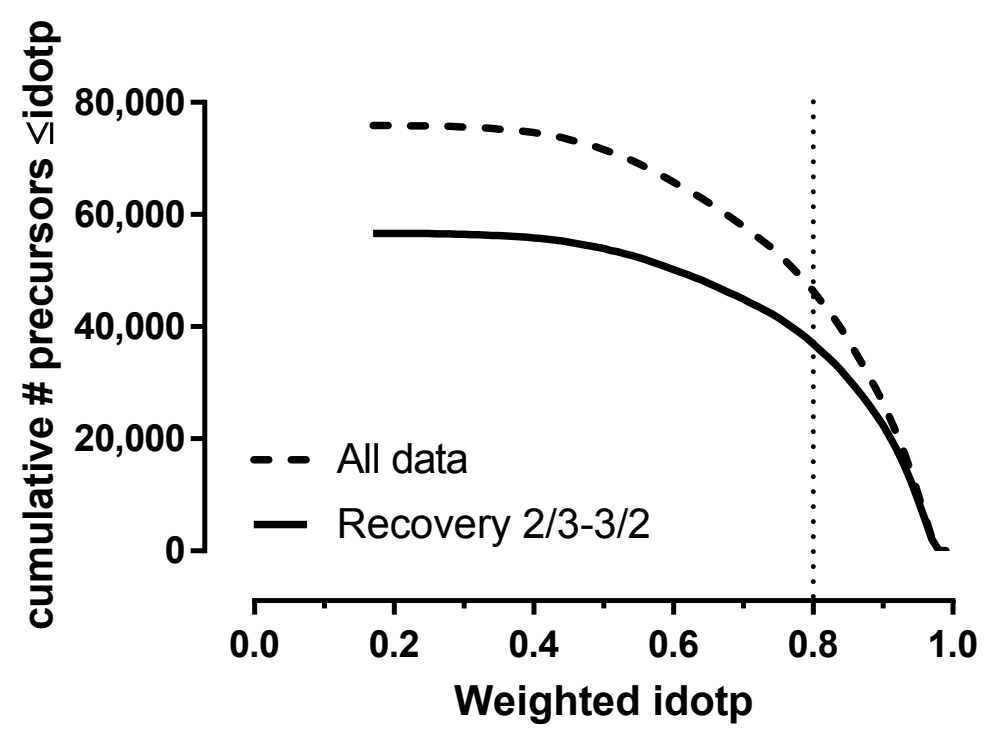

Fig. S1. Distribution of precursors with weighted idotp. Weighted idotp is defined as: sum ((idotp x intensity) of precursor per run)/ sum (precursor intensities in all runs). Solid line plot represents precursors with acceptable recoveries while dashed line plot includes all recoveries. Vertical dashed line represents the acceptable weighted idotp threshold of 0.8 . 


\begin{tabular}{llll}
\hline Mitochondria & $\square$ Peroxisome & $\diamond$ Golgi & $\circ$ Cytosol \\
$\circ$ Lysosome & $\nabla$ ER & $\diamond$ Plasma membrane $\square$ Nucleus
\end{tabular}
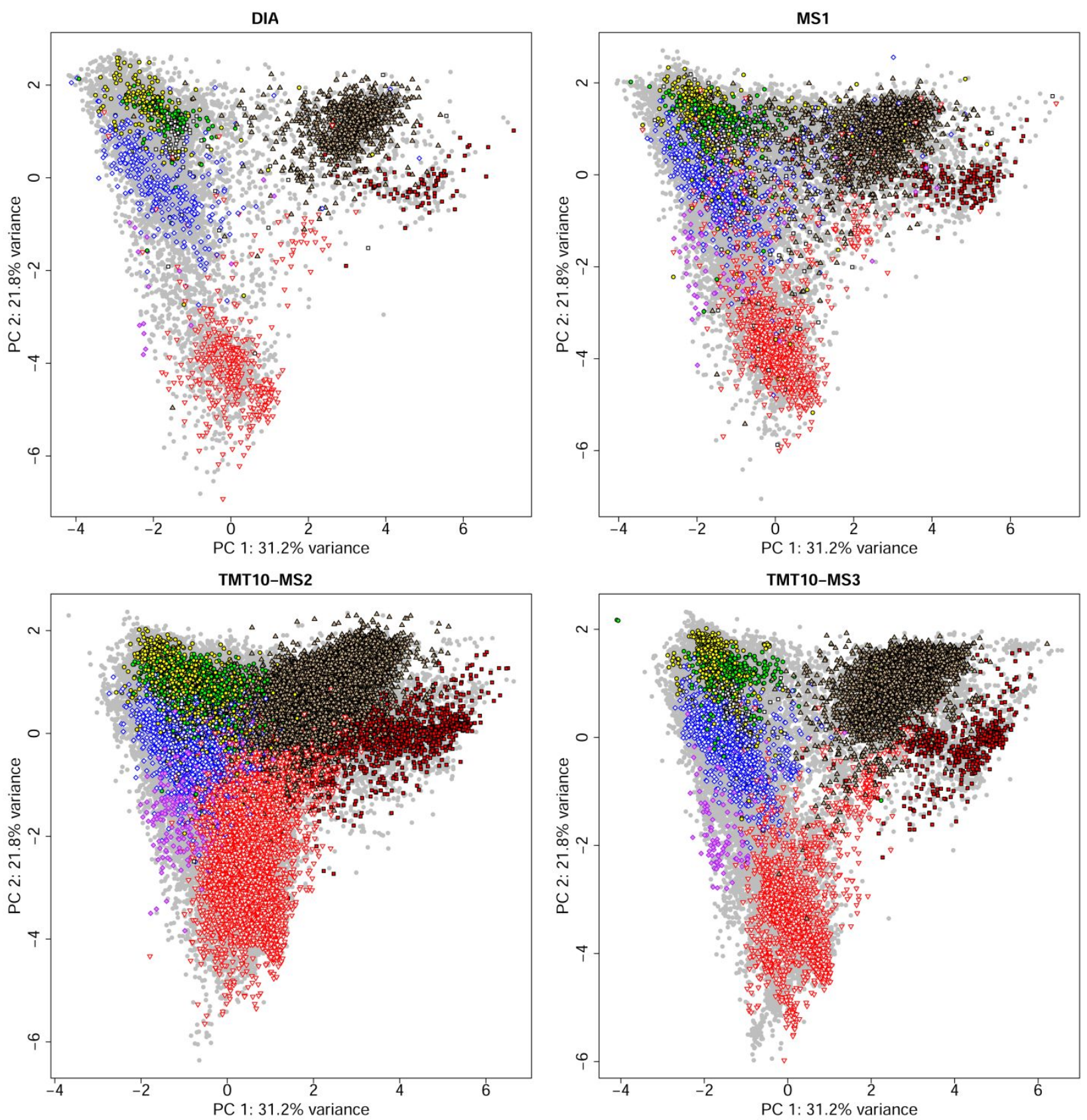

Fig. S2. Principal component 1 versus 2 of peptides obtained using the four different quantitative MS approaches. Peptides are assigned to organelles as indicated by color - unassigned peptides are indicated in grey. 


$\begin{array}{llll}\triangle \text { Mitochondria } & \because \text { Peroxisome } & \diamond \text { Golgi } & \circ \text { Cytosol } \\ \circ \text { Lysosome } & \nabla \text { ER } & \diamond \text { Plasma membrane } \square \text { Nucleus }\end{array}$
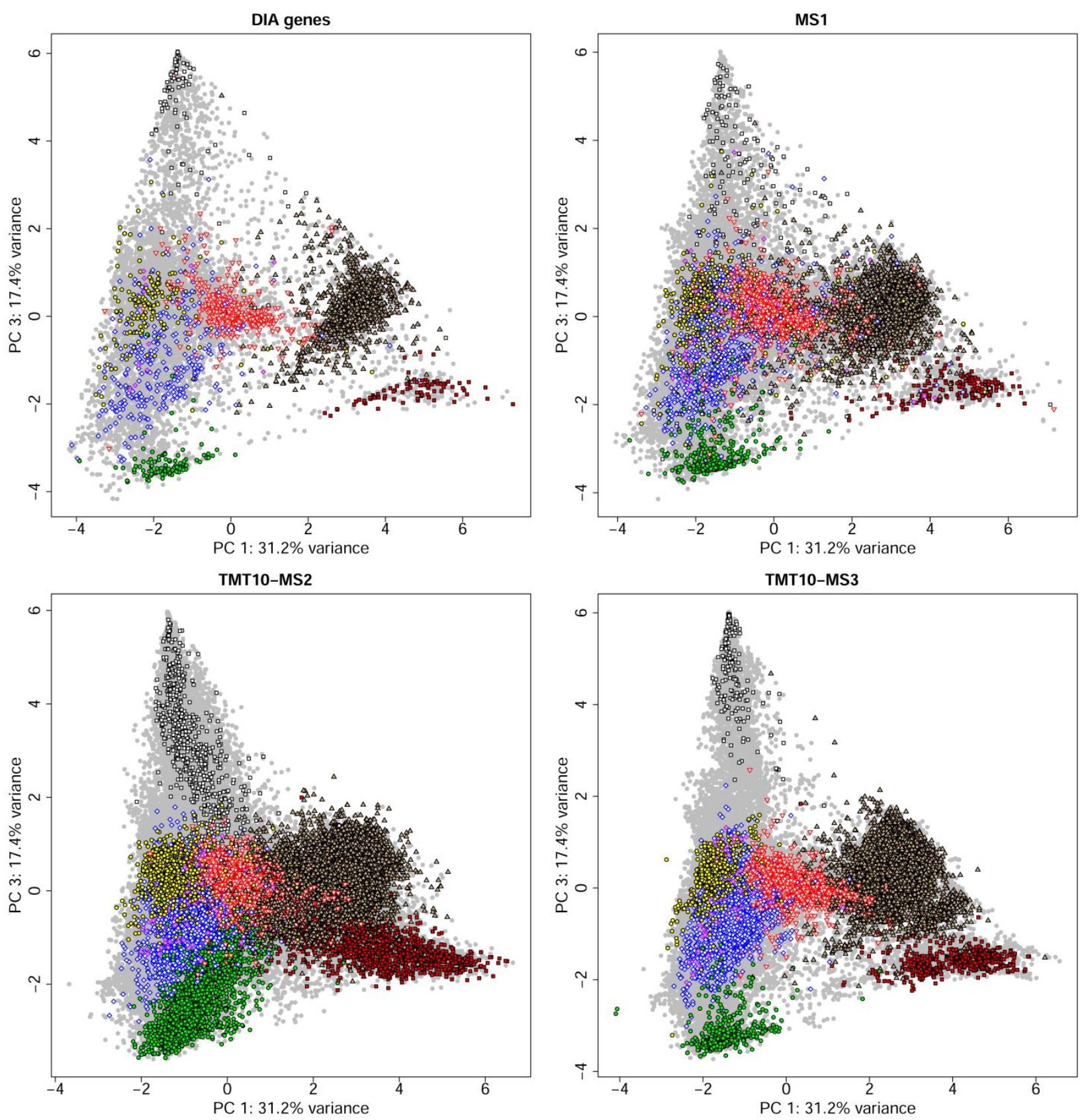

Fig. S3. Principal component 1 versus 3 of peptides obtained using the four different quantitative MS approaches. Peptides are assigned to organelles as indicated by color - unassigned peptides are indicated in grey. 


\begin{tabular}{|c|c|c|c|}
\hline $\begin{array}{l}\triangle \text { Mitochondria } \\
\text { - Lysosome }\end{array}$ & $\begin{array}{l}- \text { Peroxisome } \\
\nabla \text { ER }\end{array}$ & $\begin{array}{l}\diamond \text { Golgi } \\
\diamond \text { Plasma membrane }\end{array}$ & $\begin{array}{l}\text { - Cytosol } \\
\text { - Nucleus }\end{array}$ \\
\hline
\end{tabular}
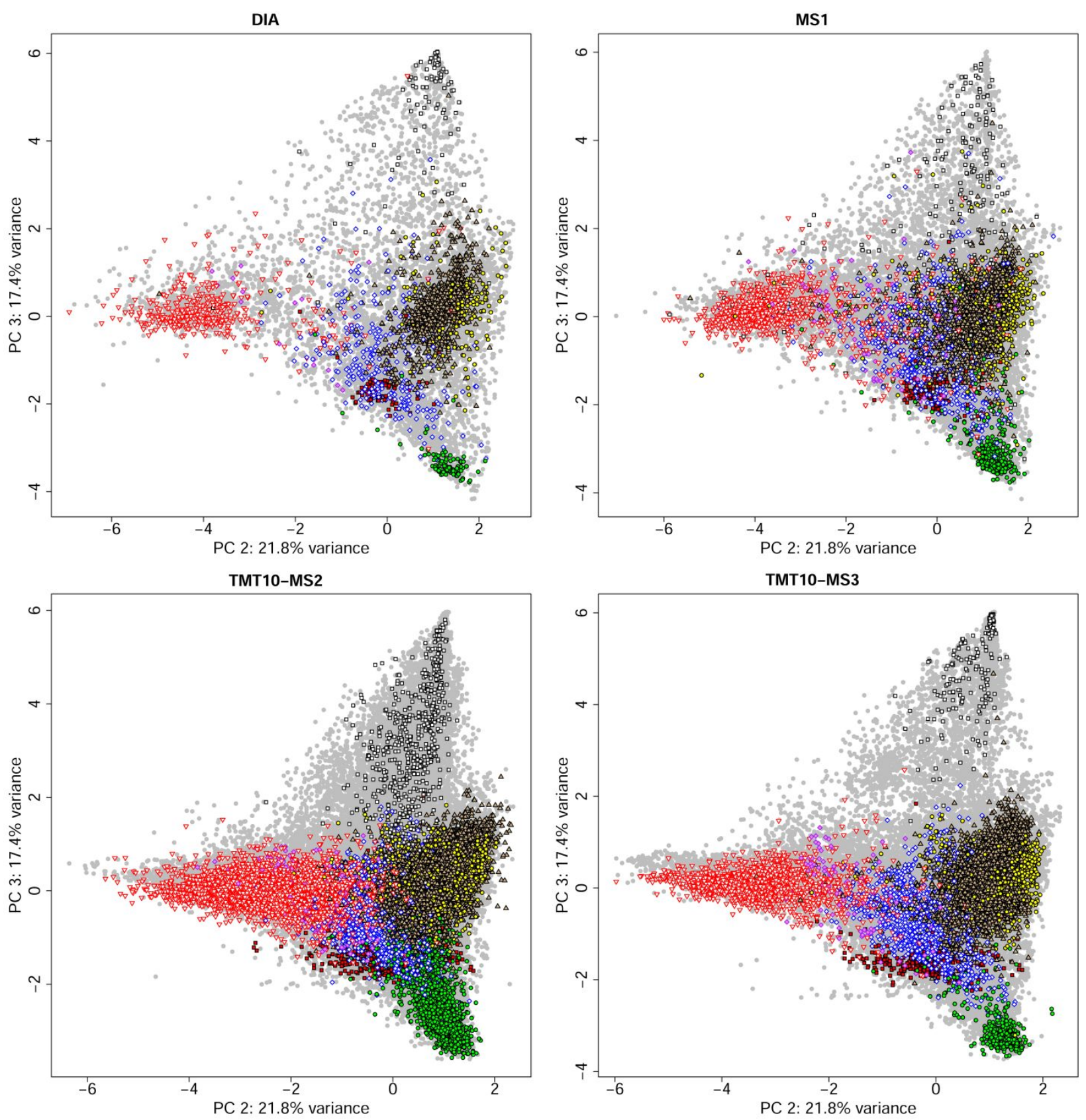

Fig. S4. Principal component 2 versus 3 of peptides obtained using the four different quantitative MS approaches. Peptides are assigned to organelles as indicated by color - unassigned peptides are indicated in grey. 